Original Article

\title{
Effects of sudden unexpected mechanical perturbation training aimed at the primary prevention of inversion ankle sprain on reactiveness of ankle movement and cortical activity in normal young adults
}

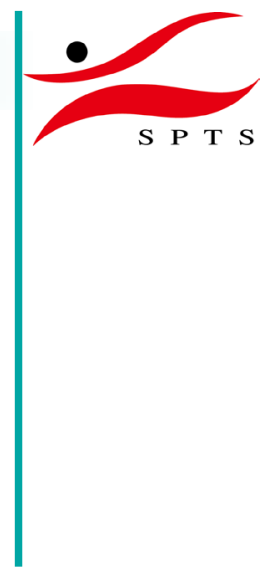

\author{
Kaori OhtA, RPT, MS ${ }^{1,2)}$, TeIJI KImUra, RPT, $\mathrm{PhD}^{3)^{*}}$ \\ 1) Graduate School of Medicine, Shinshu University: 3-1-1 Asahi, Matsumoto City, Nagano 390-8621, \\ Japan \\ 2) Omachi Municipal General Hospital, Japan \\ 3) School of Health Sciences, Faculty of Medicine, Shinshu University, Japan
}

\begin{abstract}
Purpose] To determine the effects of sudden unexpected mechanical perturbation training aimed at the primary prevention of inversion ankle sprain on the reactiveness of ankle eversion movement and cortical activity. [Participants and Methods] Sixty healthy participants were randomly allocated equally into 1) the sudden unexpected mechanical perturbation training group, 2) self-paced training group, or 3) control group. The first two groups performed each course of training 3 days per week for 4 weeks. During pre-training and post-training, the latency to peak amplitude on the surface electromyography of the peroneus longus and the time to reposition the plate back to its initial position under the right foot after sudden unexpected mechanical perturbation were measured. Functional near-infrared spectroscopy was used to measure the changes in the concentration of oxygenated hemoglobin. [Results] The latency to peak amplitude was significantly shorter in group 1 than in group 2; time to reposition the plate was the shortest among the 3 groups during post-training. The changes in the concentration of oxygenated hemoglobin were significantly increased in the supplementary motor and pre-motor areas during posttraining than during pre-training in group 1. [Conclusion] Sudden unexpected mechanical perturbation training may facilitate the primary prevention of inversion ankle sprain via the positive effects on the reactiveness of ankle eversion movement and cortical activity.

Key words: Sudden unexpected mechanical perturbation training, Inversion ankle sprain, Primary prevention
\end{abstract}

(This article was submitted Dec. 1, 2018, and was accepted Dec. 17, 2018)

\section{INTRODUCTION}

Ankle sprain is one of the most common injuries in the field of sports ${ }^{1)}$; in particular, inversion ankle sprain (IAS) occurs most frequently ${ }^{2-4)}$. It has been indicated that the primary problems after acute IAS are pain, swelling, stiffness, and dysfunction of the ankle joint ${ }^{4,5)}$. Moreover, the secondary problems of IAS are not only a long time to return to athletic participation but also an increased risk of re-injury due to instability of the ankle joint ${ }^{4,6-8)}$. Therefore, the primary prevention of IAS has become very important in order to protect sports players from the occurrence of those primary and secondary problems of $\mathrm{IAS}^{8)}$.

Regarding the effect of intervention for the primary prevention of ankle sprain, previous systematic reviews indicated

*Corresponding author. Teiji Kimura (E-mail: tkimura@shinshu-u.ac.jp)

(C2019 The Society of Physical Therapy Science. Published by IPEC Inc.

(c) (1) $\odot$ This is an open-access article distributed under the terms of the Creative Commons Attribution Non-Commercial No Derivatives CC. ${ }_{\text {BY }}$ ND (by-nc-nd) License. (CC-BY-NC-ND 4.0: https://creativecommons.org/licenses/by-nc-nd/4.0/) 
that there is inconclusive evidence that external ankle support ${ }^{9)}$, ankle taping or ankle braces ${ }^{10)}$, and proprioceptive training including exercise involving balancing on an unstable surface such as an ankle disk or wobble board ${ }^{11)}$ reduce the number of initial ankle sprains. A small number of previous studies involving subjects with no history of ankle sprain and a wide confidence interval of pooled results ${ }^{11)}$ has been published. On the contrary, previous systematic reviews regarding the effect of intervention for prevention of recurrence of ankle sprain reported that an external ankle support in the form of a semi-rigid ankle orthosis or Aircast brace ${ }^{9)}$, ankle taping or several kinds of ankle braces ${ }^{10)}$, and proprioceptive training with an ankle disk or wobble board ${ }^{11)}$ show good evidence for reducing the occurrence rate of ankle sprain among participants with a history of ankle sprain. The effectiveness of an ankle support, comprising an ankle brace and ankle taping, in prevention of recurrence was revealed in previous systematic reviews ${ }^{9-11)}$; however, it has been noted that an ankle brace might be disadvantageous as a hindering element of athletic performance $8,9,12,13)$, and taping needs to be skillfully applied, as it loosens with activity and can cause skin irritation ${ }^{13)}$. As an alternative to ankle support and ankle taping for preventing ankle sprain, it has been suggested that proprioceptive training with an ankle disk or wobble board may avoid those disadvantages ${ }^{11,13)}$. Proprioceptive training might enhance the protective reaction against perturbation both peripherally and centrally ${ }^{14-16)}$, but it has been pointed out that proprioceptive training is unlikely suitable for preventing IAS. The reason why proprioceptive training involves slower perturbation than the actual situation of sudden IAS is so one can predict perturbation from devices such as an ankle disk ${ }^{15,17}$. Further, training with more rapid joint loading under time-critical conditions similar to impending ankle sprain ${ }^{15}$ and training with sudden unexpected perturbation ${ }^{17)}$ have been recommended. Therefore, it was thought that clarifying the effect of sudden unexpected mechanical perturbation (SUMP) training by using a mechanical perturbation device, which can control the angle and angle velocity of perturbation in order to simulate the condition of IAS within the safety margin, may be a more effective approach for the primary prevention of IAS.

As an evaluation method to clarify the effect of training aimed at the primary prevention and prevention of recurrence of IAS, previous studies have analyzed the reaction time of peroneal muscles or actual eversion movement against perturbation toward ankle inversion by using both a trap door, which was constructed by an apparatus with a tilting foot support, and a tilting platform, which was constructed mostly of two independent movable trap doors that dropped in inversion in the frontal plane ${ }^{18,19)}$. The measurements obtained from using a trap door and tilting platform can be used to evaluate the role of the dynamic defense mechanism of the ankle joint against suddenly forced inversion. However, regarding the validity of the measurement, it was pointed out that the distribution of body weight on these types of equipment might influence the angle velocity of perturbation toward ankle inversion and the peroneus reaction time ${ }^{18,20}$. Thus, it is necessary to control the angle velocity of perturbation among the participants. Moreover, the importance of the dynamic defense mechanism of ankle eversion by the peroneal muscles against suddenly forced inversion to prevent ankle inversion injuries including IAS has been indicated ${ }^{14,21)}$, but there has been no report about the analysis of reactiveness of ankle eversion to SUMP with a controlled angle and angle velocity. Therefore, it was thought that the quantitative measurement of reactiveness of ankle eversion movement to mechanically controlled sudden unexpected perturbation may be an effective approach to clarify the effect of SUMP training on the primary prevention of IAS.

Additionally, it was thought that perturbation training ${ }^{17}$ ) and the measurement of reactiveness of ankle eversion movement against sudden perturbation ${ }^{14)}$ might involve not only a peripheral response but also the central neural system. Combined measurement of the reaction time of peroneal muscles or actual eversion movement against perturbation and cortical activity, which seems to be an indicator of central motor control aimed at prevention of IAS, has not been performed. Previous studies have analyzed the specificity of cortical activity in central motor control and used functional near-infrared spectroscopy (fNIRS) in balance control-related reactive movement to perturbation ${ }^{22-24)}$. Although these previous studies reported that there were perturbation-induced signal change in a portion of the cortical area ${ }^{23)}$ and correlations between some of the cortical area and sway direction ${ }^{24)}$, the longitudinal change of cortical activity using fNIRS with perturbation training for the primary prevention of IAS has not been investigated. Thus, it was thought that measurements of cortical activity combined with reactiveness of ankle eversion movement were valid in the evaluation of the effect of SUMP training on both the peripheral protective response and central motor control.

For these reasons, it was hypothesized that SUMP training for the primary prevention of IAS would lead to changes in both reactiveness of ankle eversion movement and cortical activity.

The effects of SUMP training on reactiveness of ankle eversion movement and cortical activity in normal young adults without a history of IAS have not been investigated using a mechanical perturbation device that can control the angle velocity and range of perturbation.

Therefore, the purpose of this study was to clarify the effect of SUMP training aimed at the primary prevention of inversion ankle sprain on the reactiveness of ankle eversion movement and cortical activity by using a custom-made perturbation device that can control the angle and angle velocity of perturbation toward the ankle inversion direction, simulating IAS in normal young adults without a history of IAS.

\section{PARTICIPANTS AND METHODS}

Sixty healthy individuals ( 24 males and 36 females) with no history of ankle sprain that required medical care within several years, without other lower extremity injuries, and with right-foot dominance (according to criteria of the Chapman 
dominant test) were recruited from the university community to participate in this study. Their age, height, and weight were $20.9 \pm 2.0$ years, $163.2 \pm 7.8 \mathrm{~cm}$, and 56.1 $\pm 6.6 \mathrm{~kg}$, respectively (mean \pm standard deviation [SD]). No participants performed continuous ankle training such as ankle eversion training using an elastic band. The purpose and procedure of the study were explained to all participants, and then their written informed consent was obtained. This study was approved by the ethics committee of the School of Medicine, Shinshu University (approval number: 2068) and adhered to the ethical principles of the Declaration of Helsinki.

The present study was designed as a randomized controlled trial with a 4-week training period, and measurements were obtained during pre-training and post-training periods. Sixty participants were randomly allocated equally into 3 groups as follows by a table of random numbers: 1) SUMP training group, 2) self-paced (SP) training group, and 3) control group with no training. Participants in the SUMP and SP training groups performed each type of training 3 days per week for 4 weeks; the control group did not perform any training, but they participated in the measurements obtained at pre-training and post-training. At pre-training and post-training, reactiveness of ankle eversion movement and cortical activity were measured when participants reacted to SUMP toward the ankle inversion direction.

With regard to equipment, a custom-made perturbation device (Tanigawa Corp., Nagano, Japan) was used to generate SUMP toward the ankle inversion direction for SUMP training and to obtain the measurements of cortical activity and reactiveness of ankle eversion movement (Fig. 1A, B). The perturbation device possessed a plate (width $15 \mathrm{~cm}$, length $35 \mathrm{~cm}$, height from floor approximately $15 \mathrm{~cm}$ ) that was controlled by a controller connected to a servo system (servo amplifier SV-040P1, servo motor SV-M040AK; KEYENCE Corp., Osaka, Japan). The plate generated actual perturbation and was set under participants' right foot while they maintained a quiet standing position. Additionally, the plate could generate SUMP toward the ankle inversion direction with an optimal range of angle and angle velocity at a random interval, and it was able to output an analog signal of the plate movement. Further, this perturbation device was able to control the angle and angle velocity of the plate movement without affecting any participant's body weight, which made it different from previously used devices such as the trap door and tilting platform. Similar to previous studies ${ }^{21,25-27)}$, the axis of rotation of the plate under the right foot corresponded to the subtalar joint. Maximal range of the plate tilting to the inversion direction was set at $30^{\circ}$, which was confirmed as the safety range of motion, according to many similar previous studies ${ }^{18-20)}$ and our pilot study. Furthermore, the angle velocity of the plate tilting toward the ankle inversion direction was set at $300^{\circ} / \mathrm{sec}$, which was also confirmed as the safety angle velocity and as the optimal angle velocity that simulates actual IAS, according to similar previous studies $^{14,18,19,21)}$ and our pilot study. Perturbation was generated by the actuator of the perturbation device, which can control both the angle and angle velocity by the plate controller. In consideration of safety, a stainless steel wall was set around the right foot to prevent the right foot from slipping on the plate. As soon as the plate tilted to $30^{\circ}$, the function of the plate controller by the actuator was changed to off, and then the plate returned movement by the participant from $30^{\circ}$ to $0^{\circ}$ without mechanical resistance by switching off the actuator; these movements were initiated by a program for the plate controller in order to simulate actual IAS. Although the terminal angle of the plate movement for mechanical perturbation was controlled at $30^{\circ}$ by the plate controller, a mechanical stopper was also set under the plate to prevent overtilting from exceeding $30^{\circ}$. An analog signal of plate movement, which indicates reactiveness of ankle eversion movement by the participant, was outputted from plate controller of the perturbation device to a personal computer for data analysis.

As for measurements protocols during pre-training and post-training, participants stood barefoot on the perturbation device with their feet $15 \mathrm{~cm}$ apart with reference to their average stride width during walking ${ }^{28}$, and they were instructed to equally distribute their body weight on their feet. Then, they were instructed to react to the repositioning of the plate back to its initial position as fast as possible when feeling the plate movement toward the ankle inversion direction. To exclude prediction of 


\begin{tabular}{|c|c|c|c|c|c|c|c|c|c|c|c|c|c|c|c|c|}
\hline & \multirow[t]{2}{*}{ (1) } & \multirow[t]{2}{*}{1} & (1) & 2 & (2) & 3 & $\mathrm{Cl}$ & 4 & (3) & 5 & (3) & 6 & (4) & & \\
\hline & & & & 8 & & 9 & & 10 & & 11 & & 12 & & 13 & & \\
\hline (5) & 14 & (4) & 15 & (6) & 16 & (5) & 17 & (7) & 18 & (6) & 19 & (8) & 20 & (7) & 21 & (9) \\
\hline 22 & & 23 & & 24 & & 25 & & 26 & & 27 & & 28 & & 29 & & 30 \\
\hline (8) & 31 & (10) & 32 & (9) & 33 & (11) & 34 & (10) & 35 & (12) & 36 & (11) & 37 & (13) & 38 & (12) \\
\hline & & 39 & & 40 & & 41 & & 42 & & 43 & & 44 & & 45 & & \\
\hline & & (13) & 46 & (14) & 47 & (14) & 48 & (15) & 49 & (15) & 50 & (16) & 51 & (16) & & \\
\hline
\end{tabular}

Fig. 2. The location of each optode and channel.

The schema represents the locations of optodes and channels (gray circle: light source, white circle: detector, white quadrate: channels, Cz: vertex).

the timing of perturbation, participants were instructed to maintain a quiet standing position without anticipating the timing of the plate tilting and watching the front white wall. At first, SUMP toward the ankle inversion direction was provided to the right foot at intervals randomized between 10 and $15 \mathrm{~s}$ over the 10 trials. While participants reacted to the perturbation, surface electromyography (sEMG) of the right peroneus longus and plate movement, as indicators of reactiveness of ankle movement, and cortical activity, as an indicator of central motor control, were measured over the 10 trials. Reactive muscle activity of the right peroneus longus was recorded by using a telemetric sEMG system (TeleMyo 2400T G2 EM-602; Noraxon Inc., Scottsdale, AZ, USA). After skin preparation for the sEMG recording, bipolar Ag/AgCl surface disposable electrodes (Vitrode M-150; Nihon Kohden Corp., Tokyo, Japan) were placed with a center-to-center distance of $3 \mathrm{~cm}$ apart, parallel to the muscle belly of the peroneus longus and the proximal third of the lower leg. To detect the cortical activity during reactive ankle movement, 51-channel (ch) fNIRS (FOIRE-3000; Shimadzu Corp., Kyoto, Japan) with 16 light sources and 16 detectors was used at a sampling interval of $250 \mathrm{~ms}$ (Fig. 2). The optodes were attached to the skull surface with a flexible adjustable surface holder (FLASH; Shimadzu Corp.) at an inter-optode distance of $3 \mathrm{~cm}$. The optodes were placed on the frontoparietal skull surface based on the international 10-20 system to cover the primary motor area, pre-motor area, supplementary motor area, and prefrontal area, according to a previous study ${ }^{29)}$. The channels were defined as the midpoint of the corresponding light source-detector pair (Fig. 2). As an indicator of cortical activity during reactive ankle movement to the mechanical perturbation, concentration changes in the oxygenated hemoglobin (oxy-Hb) level were measured. Regarding the anatomical information, the location of the optodes was recorded by the 3-dimensional digitizer (FASTRAK; Polhemus, Inc., Colchester, VT, USA) to estimate the locations of the fNIRS channels.

Regarding training protocols, both participants in the SUMP and SP training groups performed 3 sets of 10 trials per day, and the training was performed 3 days per week for 4 weeks. In the 3 sets of trials, there were 30 -s rests between sets. The custom-made perturbation device controlled both the mechanical perturbation mode for SUMP training and free mode for SP training. In the SUMP training group, participants were instructed to react as fast as possible against SUMP generated toward the ankle inversion direction via the custom-made perturbation device. The range of the angle of mechanical perturbation was set at 0 to $30^{\circ}$, and angle velocity was set at $300^{\circ} / \mathrm{sec}$. Mechanical perturbation was provided at intervals randomized between 1 and $5 \mathrm{~s}$ over the 10 trials, which was different from mechanical perturbation used for measurements during pre-training and post-training. The time to reposition the plate to its initial position every trial, which was indicated on the controller's digital display, was afforded to participants orally by the examiner after every trial as feedback. Participants in the SP training group were instructed to move the plate of the custom-made perturbation device as fast as possible by self-paced movement during the 10 trials. In the SP training group, the participant could move the plate freely without mechanical resistance from 0 to $30^{\circ}$. The duration of plate movement in 10 trials, which was measured by a stopwatch, was afforded to participants orally from the examiner after every set as feedback. The feedback was meant to encourage participants to perform their best. Participants in the control group did not undergo training; they only underwent measurements at pre-training and post-training periods.

For data analysis, the signals of sEMG and plate movement were analog-to-digital converted at a sampling rate of 1,500 $\mathrm{Hz}$ and analyzed by using biosignal-analyzing software (MyoResearch XP EM-129M; Noraxon Inc.). Raw sEMG signals of the peroneus longus were band-pass filtered between 10 and $350 \mathrm{~Hz}$ and rectified, and smoothing of the sEMG signals was performed by calculating the root-mean-square (RMS) using a 50-ms moving window. Data analysis regarding reactiveness of ankle eversion movement against mechanical perturbation was performed using the following parameters: (1) latency to peak amplitude (LPA) of the RMS in sEMG of the peroneus longus and (2) time to reposition the plate (TRP) to its initial position. During pre-training and post-training, (1) LPA was analyzed according to the duration from the start of mechanical perturbation to the peak amplitude of the RMS in sEMG of the peroneus longus, and (2) TRP was analyzed according to the duration from the start of mechanical perturbation to repositioning of the plate to its initial position under the right foot. LPA and TRP were analyzed by using biosignal-analyzing software (MyoResearch XP EM-129M; Noraxon Inc.). To detect the cortical activity during reactive movement, concentration changes in the oxy-Hb level of each group were calculated by subtracting the mean value of the baseline ( $2 \mathrm{~s}$ before perturbation of the initial trial) from the mean value, which included activation peaks of oxy- $\mathrm{Hb}$ (from 3.25 to $5.25 \mathrm{~s}$ after the start of perturbation), based on previous studies.

In statistical analysis, all parameters were compared between and within groups. Since the data for LPA, TRP, and changes 


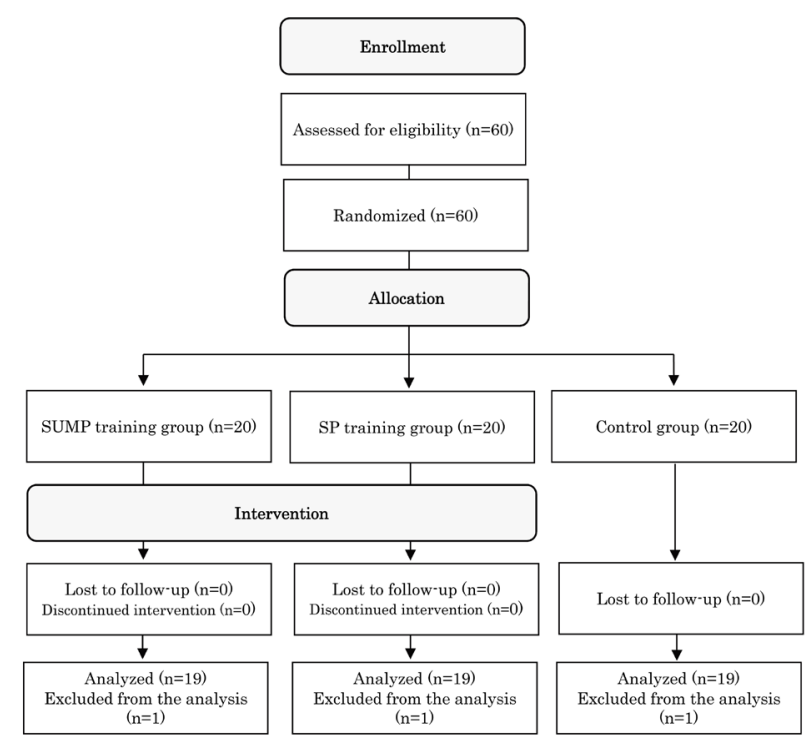

Fig. 3. CONSORT diagram of the flow of participants through the study.

SUMP: sudden unexpected mechanical perturbation.

SP: self-paced.

Table 1. Participant characteristics

\begin{tabular}{lccc}
\hline \multicolumn{1}{c}{ Variable } & SUMP training group & SP training group & Control group \\
\hline $\mathrm{n}$ (male: female, gender) & $19(7: 12)$ & $19(8: 11)$ & $19(8: 11)$ \\
Age $(\mathrm{yrs})$ & $20.8 \pm 2.0$ & $21.1 \pm 2.1$ & $20.9 \pm 2.0$ \\
Height $(\mathrm{cm})$ & $163.8 \pm 9.5$ & $164.0 \pm 7.0$ & $161.8 \pm 6.6$ \\
Weight $(\mathrm{kg})$ & $55.6 \pm 6.7$ & $56.6 \pm 5.1$ & $56.3 \pm 8.2$ \\
\hline
\end{tabular}

Data are presented as a mean \pm standard deviation.

There are no statistical differences among the 3 groups.

SUMP: sudden unexpected mechanical perturbation; SP: self-paced.

in the oxy-Hb level were not normally distributed according to the Shapiro-Wilks test, the Kruskal-Wallis test was used for inter-group comparison. If there was significance in the inter-group comparison, the Mann-Whitney U test with the Bonferroni procedure $(\mathrm{p}<0.017)$ was used as a post hoc test. The Wilcoxon signed-rank test was used to perform intra-group comparisons. Statistical significance was set at 0.05 , and statistical analysis was performed with SPSS 18.0 for Windows (SPSS Inc., Chicago, IL, USA).

\section{RESULTS}

The flow of participants through the study is detailed in accordance with the CONSORT statement (Fig. 3). One male participant in the SUMP training group was excluded from data analysis since the participant was unable to perform reactive ankle movement precisely. Two female participants (1 each in the SP and control groups) were excluded from data analysis since their TRP value exceeded the average $\pm 3 \mathrm{SD}$ reported in a previous study ${ }^{30)}$. The participant characteristics, excluding these 3 participants, are shown in Table 1. There were no significant differences among the 3 groups in participant characteristics. In the data analysis of changes in the oxy-Hb level, the data of oxy- $\mathrm{Hb}$ in $9 \mathrm{ch}, 11 \mathrm{ch}$, and $27 \mathrm{ch}$ were excluded from statistical analysis, because large artifacts were present in 16 participants.

The inter-group comparison of each parameter is shown in Table 2. A significant difference in LPA was observed among the 3 groups during post-training $(\mathrm{p}<0.05)$, and LPA was significantly shorter in the SUMP training group than in the SP training group $(\mathrm{p}<0.017$, post hoc test). With regard to TRP, a significant difference was observed among the 3 groups during post-training $(\mathrm{p}<0.05)$. TRP was faster in the SUMP training group than in the SP training and control groups during post-training; however, there was no significant difference in the results of the post hoc test. There was also no significant difference among the 3 groups in concentration changes of the oxy-Hb during pre-training and post-training.

The intra-group comparison of each parameter is shown in Table 3. Regarding LPA, there were no significant differences 
Table 2. Inter-group comparisons of latency to peak amplitude (LPA) and time to reposition the plate (TRP)

\begin{tabular}{lcccc} 
LPA & & & & \\
\hline pre-training & $140.86 \pm 52.13$ & $155.37 \pm 61.12$ & $168.54 \pm 41.78$ & p value \\
post-training & $124.7 \pm 31.15 \mathrm{~b}^{*}$ & $174.86 \pm 56.72 \mathrm{~b}^{*}$ & $156.99 \pm 44.54$ & $\mathrm{a}^{*}$ \\
\hline
\end{tabular}

Data are presented as a mean \pm standard deviation (ms).

SUMP: sudden unexpected mechanical perturbation; SP: self-paced.

a: Kruskal-Wallis test; ${ }^{*} \mathrm{p}<0.05$.

b: Mann-Whitney U test (Bonferroni procedure); ${ }^{*} \mathrm{p}<0.017$.

TRP

\begin{tabular}{lcccc} 
TRP & SUMP training group & SP training group & Control group & p value \\
\hline pre-training & $288.88 \pm 66.95$ & $283.01 \pm 65.77$ & $277.41 \pm 40.78$ & \\
post-training & $259.13 \pm 40.04$ & $321.72 \pm 94.44$ & $338.38 \pm 125.38$ & $\mathrm{a}^{*}$ \\
\hline
\end{tabular}

Data are presented as a mean \pm standard deviation (ms).

SUMP: sudden unexpected mechanical perturbation; SP: self-paced.

a: Kruskal-Wallis test; ${ }^{*} \mathrm{p}<0.05$.

Table 3. Intra-group comparisons of latency to peak amplitude (LPA), time to reposition the plate (TRP), and concentration changes in oxygenated hemoglobin (oxy-Hb)

\begin{tabular}{lrr} 
LPA & pre-training & post-training \\
\hline SUMP training group & $140.86 \pm 52.13$ & $124.7 \pm 31.15$ \\
SP training group & $155.37 \pm 61.12$ & $174.86 \pm 56.72$ \\
Control group & $168.54 \pm 41.78$ & $156.99 \pm 44.54$ \\
\hline
\end{tabular}

Data are presented as a mean \pm standard deviation (ms).

SUMP: sudden unexpected mechanical perturbation; SP: self-paced.

TRP

\begin{tabular}{lccc} 
& pre-training & post-training & p value \\
\hline SUMP training group & $288.88 \pm 66.95$ & $259.13 \pm 40.04$ & $\mathrm{a}^{*}$ \\
SP training group & $283.01 \pm 65.77$ & $321.72 \pm 94.44$ & \\
Control group & $277.41 \pm 40.78$ & $338.38 \pm 125.38$ & $\mathrm{a}^{*}$ \\
\hline
\end{tabular}

Data are presented as a mean \pm standard deviation (ms).

SUMP: sudden unexpected mechanical perturbation; SP: self-paced.

a: Wilcoxon signed-rank test; ${ }^{*} \mathrm{p}<0.05$

Concentration changes in oxy- $\mathrm{Hb}$

\begin{tabular}{lrccc}
\hline & ch & pre-training & post-training & p value \\
\hline SUMP training group & 19 & $-0.00188 \pm 0.01619$ & $0.00641 \pm 0.01189$ & $\mathrm{a}^{*}$ \\
SP training group & 28 & $-0.00154 \pm 0.00961$ & $0.00741 \pm 0.01189$ & $\mathrm{a}^{*}$ \\
& 19 & $0.00036 \pm 0.01819$ & $0.01053 \pm 0.01684$ & $\mathrm{a}^{*}$ \\
Control group & 41 & $0.00029 \pm 0.01262$ & $0.01027 \pm 0.01411$ & $\mathrm{a}^{*}$ \\
& 3 & $-0.00854 \pm 0.02063$ & $0.00261 \pm 0.02211$ & $\mathrm{a}^{*}$ \\
& 4 & $-0.01552 \pm 0.02259$ & $0.00312 \pm 0.02057$ & $\mathrm{a}^{*}$ \\
& 5 & $-0.00908 \pm 0.01769$ & $0.00577 \pm 0.01776$ & $\mathrm{a}^{*}$ \\
& 34 & $-0.0061 \pm 0.01456$ & $0.00035 \pm 0.01432$ & $\mathrm{a}^{*}$ \\
\hline
\end{tabular}

Data are presented as a mean \pm standard deviation $(\mathrm{mM} \cdot \mathrm{cm})$.

The table details only the channels that have statistical significance.

SUMP: sudden unexpected mechanical perturbation; SP: self-paced; ch: channel.

a: Wilcoxon signed-rank test; ${ }^{*} \mathrm{p}<0.05$

in the 3 groups between the post-training and pre-training. TRP was significantly shortened during post-training compared with during pre-training in the SUMP training group ( $<<0.05$ ), and significantly delayed during post-training compared with during pre-training in the control group $(\mathrm{p}<0.05)$. In the SUMP training group, the oxy-Hb concentration changes in the SUMP training group were significantly increased in the supplementary motor area and pre-motor area during post-training compared with during pre-training $(19 \mathrm{ch}, 28 \mathrm{ch}: \mathrm{p}<0.05)$. In the SP training group, a significant increase of the oxy-Hb concentration changes were observed in the supplementary motor area and prefrontal area during post-training (19 ch, 41 ch: $\mathrm{p}<0.05)$. In the control group, the oxy-Hb concentration changes in the primary motor cortex and prefrontal area were significantly increased during post-training $(3 \mathrm{ch}, 4 \mathrm{ch}, 5 \mathrm{ch}, 34 \mathrm{ch}: \mathrm{p}<0.05)$. 


\section{DISCUSSION}

In this study, LPA, which was thought to be an indicator of reactiveness of the peroneus longus, was significantly shortened in the SUMP training group compared to the SP training group, and TRP, which was thought to be an indicator of actual ankle eversion movement, was shortened in the SUMP training group compared to the SP training group and control group. In the intra-group comparison, the oxy-Hb concentration changes of the supplementary motor area and pre-motor area in the SUMP training group was significantly increased during post-training compared with during pre-training. Based on these results, the present study demonstrated that SUMP training might enhance the reactiveness of ankle eversion movement and cortical activity compared with SP training and no training.

In many previous studies, although the reaction time of the peroneal muscles including the peroneus longus, as detected by sEMG, has been widely used as an evaluation indicator for the prevention of ankle sprain ${ }^{19)}$, the present study used LPA and TRP as indicators in terms of the primary prevention of IAS. With regard to the peroneus reaction time as an evaluation indicator, it has been pointed out that the peroneus reaction time was too slow to prevent lateral ligament overloading from sudden unexpected inversion perturbation of the ankle ${ }^{14,31)}$. On the contrary, it was pointed out that if the ankle has become subject to inversion torque, injury can still be prevented if the reaction to counteract the inversion torque is sufficiently fast and strong, and the time from perturbation until a substantial eversion force can be produced in the appropriate muscle is far more important ${ }^{31)}$. Furthermore, regarding the mechanism of inversion ankle injury, it has been reported that most likely the laterally directed force does not produce the injury itself but causes the player to load weight with the ankle in a vulnerable, inverted position ${ }^{3)}$. Hence, IAS might be prevented if the eversion torque can be produced sufficiently fast and strong before the ankle is inverted, which is a compromising position of IAS. The present study used two kinds of indicators, LPA as an indicator of the time from onset of perturbation to substantial eversion force and TRP as an indicator of real defensive movement of reactive ankle eversion, for the prevention of IAS.

Our findings suggest that SUMP training could facilitate defensive reaction in terms of LPA and TRP to SUMP. Because significant changes of the two components that were thought to be defensive reactions, such as generation of the substantial resistive eversion force in the peroneus longus and repositioning of the plate from the inverted position, must be faster under SUMP training, it was thought that SUMP training might enhance reactiveness to prevent the ankle from being in the compromising position that induces IAS.

Regarding the positive effects of ankle eversion movement on reactiveness, a significant increase in the oxy-Hb concentration changes in areas such as the supplementary motor area and pre-motor area, which is associated with the automation of movement and movement programming, was recognized after 4 weeks of SUMP training. Concerning the involvement of the central nervous system with reactiveness to perturbation, it has been reported that perturbation training might accelerate the response of muscle spindle and neural input in an attempt to regain stability against perturbation ${ }^{17)}$, and the efficient response to sudden inversion is not a reflex reaction but at least in part a learned ability ${ }^{21)}$. Moreover, Ashton-Miller et al. ${ }^{15)}$ indicated that there was a possibility that proprioceptive training increases the attention paid to proprioceptive cues by the brain, first at the conscious level early in training and then later, after perhaps more training, at the autonomous level. Therefore, our results suggest that SUMP training might have facilitated automation of the reactive ankle eversion movement to react as quickly as possible to sudden unexpected perturbation, and further, it might have contributed to the condition that promotes the defensive reaction in LPA and TRP against mechanical perturbation.

Thus, our hypothesis that SUMP training may be an important intervention for the primary prevention of IAS may have validity.

In addition, regarding correctness of the measurements in the present study, perturbation using a custom-made perturbation device was used in order to simulate the real situation of IAS as much as possible to ensure safety and obtain a quantitative measurement.

The limitations of this study were that the effect of SUMP training on the incidence of actual IAS and the long-term training effect could not be observed for the primary prevention of IAS. In addition, it was impossible to measure the primary sensory area in the measurement of cortical activity because of the limitation of the number of optodes. Further study is required to reveal more about the effect of SUMP training on the primary prevention of IAS and whether SUMP training can contribute to decreasing the incidence of IAS, retention effect on reactiveness of ankle movement and cortical activity, and oxy-Hb concentration in the primary sensory area during reactive ankle eversion movement.

In conclusion, SUMP training and the quantitative measurement system using the custom-made perturbation device may provide new scientific evidence, as SUMP training seemed to be an effective training method for the primary prevention of IAS.

\section{Funding}

This study was funded by a research grant from the Adaptable and Seamless Technology transfer Program provided by the Japan Science and Technology Agency. 


\section{Conflict of interest}

There are no conflicts of interest.

\section{REFERENCES}

1) Waterman BR, Owens BD, Davey S, et al.: The epidemiology of ankle sprains in the United States. J Bone Joint Surg Am, 2010, 92: 2279-2284. [Medline] [CrossRef]

2) Garrick JG: The frequency of injury, mechanism of injury, and epidemiology of ankle sprains. Am J Sports Med, 1977, 5: 241-242. [Medline] [CrossRef]

3) Andersen TE, Floerenes TW, Arnason A, et al.: Video analysis of the mechanisms for ankle injuries in football. Am J Sports Med, 2004, 32: 69S-79S. [Medline] [CrossRef]

4) Fong DT, Hong Y, Chan LK, et al.: A systematic review on ankle injury and ankle sprain in sports. Sports Med, 2007, 37: 73-94. [Medline] [CrossRef]

5) Dubin JC, Comeau D, McClelland RI, et al.: Lateral and syndesmotic ankle sprain injuries: a narrative literature review. J Chiropr Med, 2011, 10: 204-219. [Medline] [CrossRef]

6) Freeman MA, Dean MR, Hanham IW: The etiology and prevention of functional instability of the foot. J Bone Joint Surg Br, 1965, 47: 678-685. [Medline] [CrossRef]

7) Tropp H, Askling C, Gillquist J: Prevention of ankle sprains. Am J Sports Med, 1985, 13: 259-262. [Medline] [CrossRef]

8) Osborne MD, Rizzo TD Jr: Prevention and treatment of ankle sprain in athletes. Sports Med, 2003, 33: 1145-1150. [Medline] [CrossRef]

9) Handoll HH, Rowe BH, Quinn KM, et al.: Interventions for preventing ankle ligament injuries. Cochrane Database Syst Rev, 2001, 3: CD000018. [Medline]

10) Dizon JM, Reyes JJ: A systematic review on the effectiveness of external ankle supports in the prevention of inversion ankle sprains among elite and recreational players. J Sci Med Sport, 2010, 13: 309-317. [Medline] [CrossRef]

11) Schiftan GS, Ross LA, Hahne AJ: The effectiveness of proprioceptive training in preventing ankle sprains in sporting populations: a systematic review and meta-analysis. J Sci Med Sport, 2015, 18: 238-244. [Medline] [CrossRef]

12) Cordova ML, Scott BD, Ingersoll CD, et al.: Effects of ankle support on lower-extremity functional performance: a meta-analysis. Med Sci Sports Exerc, 2005, 37: 635-641. [Medline] [CrossRef]

13) Verhagen E, van der Beek A, Twisk J, et al.: The effect of a proprioceptive balance board training program for the prevention of ankle sprains: a prospective controlled trial. Am J Sports Med, 2004, 32: 1385-1393. [Medline] [CrossRef]

14) Konradsen L, Voigt M, Højsgaard C: Ankle inversion injuries. The role of the dynamic defense mechanism. Am J Sports Med, 1997, 25: 54-58. [Medline] [CrossRef]

15) Ashton-Miller JA, Wojtys EM, Huston LJ, et al.: Can proprioception really be improved by exercises? Knee Surg Sports Traumatol Arthrosc, 2001 , 9: $128-136$. [Medline] [CrossRef]

16) Yong MS, Lee YS: Effect of ankle proprioceptive exercise on static and dynamic balance in normal adults. J Phys Ther Sci, 2017, 29: 242-244. [Medline] [CrossRef]

17) Taylor JB: Lower extremity perturbation training. Strength Condit J, 2011, 33: 76-83. [CrossRef]

18) Menacho MO, Pereira HM, Oliveira BI, et al.: The peroneus reaction time during sudden inversion test: systematic review. J Electromyogr Kinesiol, 2010, 20: 559-565. [Medline] [CrossRef]

19) Ha SC, Fong DT, Chan KM: Review of ankle inversion sprain simulators in the biomechanics laboratory. Asia Pac J Sports Med Arthrosc Rehabil Technol, 2015, 2: 114-121. [Medline]

20) Benesch S, Pütz W, Rosenbaum D, et al.: Reliability of peroneal reaction time measurements. Clin Biomech (Bristol, Avon), 2000, 15: 21-28. [Medline] [CrossRef]

21) Konradsen L, Peura G, Beynnon B, et al.: Ankle eversion torque response to sudden ankle inversion Torque response in unbraced, braced, and pre-activated situations. J Orthop Res, 2005, 23: 315-321. [Medline] [CrossRef]

22) Jacobs JV, Horak FB: Cortical control of postural responses. J Neural Transm (Vienna), 2007, 114: 1339-1348. [Medline] [CrossRef]

23) Mihara M, Miyai I, Hatakenaka M, et al.: Role of the prefrontal cortex in human balance control. Neuroimage, 2008, 43: 329-336. [Medline] [CrossRef]

24) Herold F, Orlowski K, Börmel S, et al.: Cortical activation during balancing on a balance board. Hum Mov Sci, 2017, 51: 51-58. [Medline] [CrossRef]

25) Ashton-Miller JA, Ottaviani RA, Hutchinson C, et al.: What best protects the inverted weightbearing ankle against further inversion? Evertor muscle strength compares favorably with shoe height, athletic tape, and three orthoses. Am J Sports Med, 1996, 24: 800-809. [Medline] [CrossRef]

26) Fuller EA: Center of pressure and its theoretical relationship to foot pathology. J Am Podiatr Med Assoc, 1999, 89: 278-291. [Medline] [CrossRef]

27) Hertel J: Functional anatomy, pathomechanics, and pathophysiology of lateral ankle instability. J Athl Train, 2002, 37: 364-375. [Medline]

28) Whittle MW: Gait analysis: an introduction. 4th ed. Edinburgh: Butterworth-Heinemann, 2007, p 120.

29) Okamoto M, Dan H, Sakamoto K, et al.: Three-dimensional probabilistic anatomical cranio-cerebral correlation via the international 10-20 system oriented for transcranial functional brain mapping. Neuroimage, 2004, 21: 99-111. [Medline] [CrossRef]

30) Miller J: Reaction time analysis with outlier exclusion: bias varies with sample size. Q J Exp Psychol A, 1991, 43: 907-912. [Medline] [CrossRef]

31) Lephart SM, Fu FH: Proprioception and neuromuscular control in joint stability. Champaign: Human Kinetics, 2000, pp 237-246. 